\title{
A laboratory study of ion-induced erosion of ice-covered carbon grains
}

\author{
T. Sabri ${ }^{1}$, G. A. Baratta ${ }^{2}$, C. Jäger ${ }^{1}$, M. E. Palumbo ${ }^{2}$, T. Henning ${ }^{1,3}$, G. Strazzulla ${ }^{2}$, and E. Wendler ${ }^{4}$ \\ ${ }^{1}$ Laboratory Astrophysics Group of the Max Planck Institute for Astronomy at the Friedrich Schiller University Jena, \\ Institute of Solid State Physics, Helmholtzweg 3, 07743 Jena, Germany \\ e-mail: tolou.sabri@uni-jena.de \\ 2 INAF-Osservatorio Astrofisico di Catania, via Santa Sofia 78, 95123 Catania, Italy \\ e-mail: Giuseppe.Baratta@oact.inaf.it \\ 3 Max-Planck-Institut für Astronomie, Königstuhl 17, 69117 Heidelberg, Germany \\ ${ }^{4}$ Institute of Solid State Physics, Friedrich Schiller University Jena, Helmholtzweg 3, 07743 Jena, Germany
}

Received 13 October 2014 / Accepted 15 December 2014

\begin{abstract}
Context. It has been confirmed that solid carbon dioxide $\left(\mathrm{CO}_{2}\right)$ is abundantly present along the line of sight to quiescent clouds and star-forming regions via space IR observations with ISO-SWS and Spitzer Space Telescope. Since $\mathrm{CO}_{2}$ has low abundance in the gas-phase, the assumption is that it is synthesized on grains after energetic processing of icy mantles and surface reactions.

Aims. The role of solid carbon is investigated as a reservoir for molecule formation and structural modifications of the material with and without an ice layer upon ion bombardment.

Methods. A gas-phase condensation technique was used to prepare a layer of ${ }^{13} \mathrm{C}$ amorphous grains. These grains were covered with $\mathrm{H}_{2} \mathrm{O}$ and $\mathrm{O}_{2}$ ice and finally bombarded with $200 \mathrm{keV}$ protons. The formation of new molecular species was analyzed using IR spectroscopy. The formation cross sections of solid ${ }^{13} \mathrm{CO}$ and ${ }^{13} \mathrm{CO}_{2}$ were determined from the increase in the column density as a function of the fluence. In addition, bare carbon grains were bombarded with a comparable fluence of protons to study the processing of the grains without ice layer. Imaging techniques such as transmission electron microscopy were used to monitor the changes in the structure.

Results. $\mathrm{CO}$ and $\mathrm{CO}_{2}$ were formed efficiently at the interface between ice and solid carbon grains at the expense of solid carbon, leading to strong grain erosion. Given the initial thickness of our C-samples (about $120 \mathrm{~nm}$ ), this resulted in an erosion of about $50 \%$ after $200 \mathrm{keV}$ proton bombardment with $6.76 \times 10^{16}$ ions $/ \mathrm{cm}^{2}$. The column density of $\mathrm{CO}$ and $\mathrm{CO}_{2}$ follows an exponential trend as a function of the irradiation fluence. The asymptotic values obtained when $\mathrm{O}_{2}$ ice is deposited on top of the carbon grains are about one order of magnitude higher than the values obtained when $\mathrm{H}_{2} \mathrm{O}$ ice is deposited on the solid carbon layer. The carbon grains were strongly graphitized upon ion bombardment in a surface layer. Less graphitization accompanied by the formation of fullerene molecules and structures from cage fragments present in the original material were observed beneath the graphitic layer.

Conclusions. The formation of $\mathrm{CO}$ and $\mathrm{CO}_{2}$ at the expense of solid carbon strongly restricts the lifetime of the solid carbon material and may influence the formation of more complex molecules in astrophysical environments. Graphitization of carbonaceous grains upon ion bombardment affect the spectral properties of the carbon grains in particular in the far-IR range.
\end{abstract}

Key words. astrochemistry - dust, extinction - ISM: clouds - molecular data

\section{Introduction}

The importance of studying the composition of icy mantles that form on dust grains in certain regions of the interstellar medium (ISM), including star-forming molecular cloud cores, is already known, which helps to unveil how such mantles affect the chemistry of the ISM, as well as the abundances of gaseous species that may freeze out onto grains (Teixeira et al. 1998; Whittet 1993; Whittet et al. 1998; Palumbo \& Strazzulla 1993; Satorre et al. 2000).

Astronomical data and studies of meteorites show that dust grains are mainly composed of silicate and carbonaceous material. They are mainly formed via gas-phase condensation in the winds of asymptotic giant branch (AGB) stars (Zhukovska \& Gail 2007; Gail et al. 2009; Groenewegen et al. 2011; Zhukovska \& Henning 2013). Carbon dust is formed in the envelopes of carbon-rich late-type stars as nanosized particles. The observational facts indicate that the chemical processing of the condensed carbon particles has to occur during the transfer from the circumstellar shells into the diffuse interstellar medium or during their stay in this medium (Jaeger et al. 1998). The evolution of carbon dust in the ISM is driven by UV photons, interaction with gas, heating processes, and ion-induced processing due to cosmic rays and to shock waves that accelerate grains, atoms, and ions. Carbonaceous components are discussed as the carrier for the $2175 \AA$ bump in the interstellar extinction curves. Small graphitic grains, hydrogenated amorphous carbon, fullerene-like carbon particles, and even polycyclic aromatic hydrocarbons are some of the possible candidates (Mennella et al. 1996; Draine 2003; Henning et al. 2004). In addition, the far-IR emission of the circumstellar environment of carbon-rich stars and dense molecular cloud cores is believed to be dominated by the emissivity of carbon dust that is assumed to be caused by graphite structures in many dust models (Draine \& Lee 1984; Li \& Greenberg 1997).

Dust grains are bare in diffuse, low-density clouds, but in dark and dense clouds, the grains are covered with ice mantles 
such as solid water doped with $\mathrm{CO}$ and $\mathrm{CO}_{2}$. Since oxygen is an abundant element, solid $\mathrm{O}_{2}$ is considered as an important constituent of icy grain mantles in dense molecular clouds (Ehrenfreund et al. 1992). However, it is difficult to detect because of its very weak absorption in the IR (Vandenbussche et al. 1999). The gaseous $\mathrm{O}_{2}$ was only detected in low abundances in dense regions of the ISM. Hincelin et al. (2011) argue that the low abundance of $\mathrm{O}_{2}$ in dark clouds can be explained by gas-grain chemical models taking the elemental oxygen depletion into account (Jenkins 2009; Whittet 2010). However, in ices, molecular oxygen is accompanied by ozone in the presence of energetic radiation (Ennis et al. 2011; Gerakines et al. 1996; Raut et al. 2011) and can be traced by $\mathrm{O}_{3}$ in ice layers on interstellar grains.

Much laboratory work has been carried out to characterize the infrared spectra of $\mathrm{CO}$ - and $\mathrm{CO}_{2}$-bearing ices of astrophysical interest and their modifications under thermal processing and irradiation (Sandford et al. 1988; Sandford \& Allamandola 1990; Palumbo \& Strazzulla 1993; Gerakines et al. 1996; Ehrenfreund et al. 1997; Palumbo \& Baratta 2000). The interaction of gas with dust grains has an important effect on the chemical evolution of the ISM. Many molecules observed in the ISM can be formed as a consequence of reactions in the ice (Herbst 2005). However, the formation of important species (e.g. $\mathrm{H}_{2}$, $\mathrm{H}_{2} \mathrm{O}$ and $\mathrm{CO}_{2}$ ) requires the presence of dust grain surfaces that act as catalysts. The ubiquitous presence of abundant carbon dioxide $\left(\mathrm{CO}_{2}\right)$ frozen on interstellar ice mantles has already been shown in several ISO and Spitzer results (de Graauw et al. 1996; Guertler et al. 1996; Whittet et al. 1998, 2007; Gerakines et al. 1999; Pontoppidan et al. 2008). Ground-based and satellitebased IR observations have clearly demonstrated the presence of frozen carbon monoxide along the line of sight to young stellar objects embedded in their parent cloud and to field stars obscured by dense molecular clouds (Tielens et al. 1991; Kerr \& Adamson 1993; Chiar et al. 1994, 1995, 1998; Teixeira et al. 1998).

$\mathrm{CO}$ is the most abundant gas phase molecule in the ISM after $\mathrm{H}_{2}$, and it can directly freeze out on refractory cores. Since $\mathrm{CO}_{2}$ is neither predicted nor observed to have considerable abundance in the gas phase in dense clouds, its condensation on interstellar grains can be negligible (Whittet \& Walker 1991). CO and $\mathrm{CO}_{2}$ may be formed as a result of energetic processing, such as UV photolysis and cosmic ray irradiation of ices containing species, such as $\mathrm{H}_{2} \mathrm{O}, \mathrm{CO}, \mathrm{CH}_{3} \mathrm{OH}, \mathrm{O}_{2}$, and $\mathrm{N}_{2}$ (Gerakines et al. 1996; Moore et al. 1991; Palumbo \& Strazzulla 1993; Strazzulla et al. 1997). Other laboratory studies have demonstrated that irradiation of solid CO (Loeffler et al. 2005) or mixtures of CO with water ice or solid $\mathrm{O}_{2}$ (Ehrenfreund et al. 1997; Gerakines et al. 2001; Palumbo et al. 1998; Satorre et al. 2000; Watanabe \& Kouchi 2002) can produce $\mathrm{CO}_{2}$. Experiments on the formation of $\mathrm{CO}$ and $\mathrm{CO}_{2}$ by energetic processing of carbonaceous surfaces covered by ice mixtures using UV photons and ions were performed by Ioppolo et al. (2009, 2013), Mennella et al. (2004, 2006), Fulvio et al. (2012), and Raut et al. (2012). Furthermore, non-energetic surface formation of $\mathrm{CO}_{2}$ directly on the interface between ice layer and grain may occur because of the reaction of species on grain cores (D'Hendecourt et al. 1985; Ioppolo et al. 2011; Noble et al. 2011).

This paper studies ion-induced interactions at the interface ice/solid in laboratory conditions that simulate the conditions in the ISM experimentally. The laboratory-produced carbon dust analogs produced from ${ }^{13} \mathrm{C}$ were covered with $\mathrm{O}_{2}$ and $\mathrm{H}_{2} \mathrm{O}$ ice layers and bombarded with $200 \mathrm{keV}$ protons. The formation of $\mathrm{CO}$ and $\mathrm{CO}_{2}$ in the ice layer and grain erosion owing to molecule

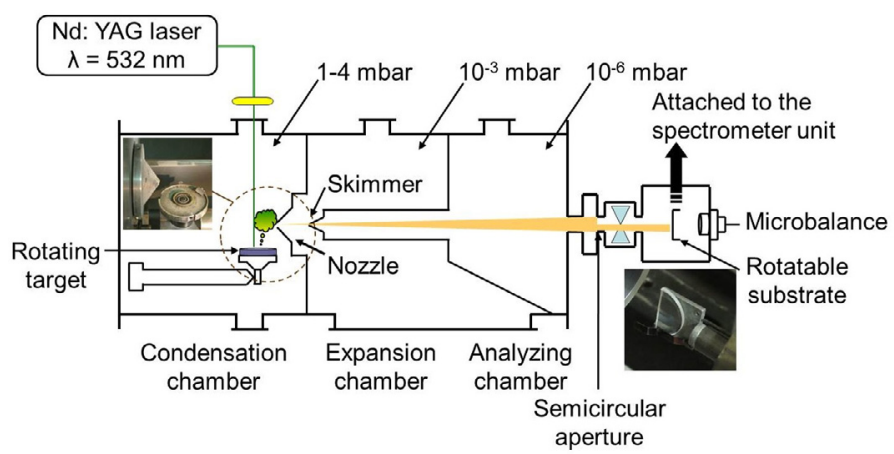

Fig. 1. Scheme of the laser ablation setup used for the condensation of graphite.

formation were observed and quantified by IR spectral analysis. In addition, sensitive analytic methods were used to investigate the strong structural and morphological modifications of the carbon grains covered by ice layers upon ion bombardment. Comparisons with structural changes of bare carbon grains upon similar ion fluences were performed.

\section{Experimental procedure}

The experiments were performed in several steps. Carbon layers were produced by laser ablation and deposited on silicon disks and on $\mathrm{KBr}$ substrates in the laboratory in Jena. In the following step, the produced carbon layers were covered with ice at the Laboratory for Experimental Astrophysics, INAF-Osservatorio Astrofisico di Catania (Italy) and processed by $\mathrm{H}^{+}$bombardment. Finally, the irradiation-induced processing of bare carbon particles was studied using the ion implanter at the Institute of Solid State Physics in Jena.

\subsection{Preparation of carbon grain samples}

The gas-phase condensation of fullerene-like nanometer-sized carbon particles was achieved by pulsed laser ablation of a rotating graphite target and subsequent condensation of the evaporated atoms, molecules, and clusters in a quenching gas of $\mathrm{He} / \mathrm{H}_{2}$ (2:1) atmosphere at a pressure of 3 mbar. The targets used for the laser evaporation were produced from isotopically pure ${ }^{13} \mathrm{C}$ powder. A scheme of the experimental setup is shown in Fig. 1. A more detailed description of the experimental procedure is given in Jäger et al. (2008) and Sabri et al. (2014). The final thickness of the deposits on either $\mathrm{KBr}$ or silicon substrates were in the range of around $120 \mathrm{~nm}$ controlled by a microbalance. The measured sample thickness obtained from the microbalance corresponds rather to a compact carbon layer. However, the layer consists of nanometer-sized carbon grains that are agglomerated resulting in a very porous carbon layer of larger thickness (see Sect. 3.3).

The laser ablation is attached to a Fourier transform infrared (FTIR) spectrometer to enable in-situ spectroscopy of the deposited grains. For this purpose, the substrate can be turned for either in-situ IR spectroscopy or for the deposition process.

\subsection{Ion bombardment of carbon grains covered by ice}

The carbon particles were covered with $\mathrm{O}_{2}$ and $\mathrm{H}_{2} \mathrm{O}$ ice and finally bombarded with $200 \mathrm{keV}$ protons. Experiments are carried out in a stainless steel ultra-high vacuum chamber with a base pressure of about $10^{-9}$ mbar as shown in Fig. 2. Inside the vacuum chamber, a $\mathrm{KBr}$ substrate covered by a particulate 


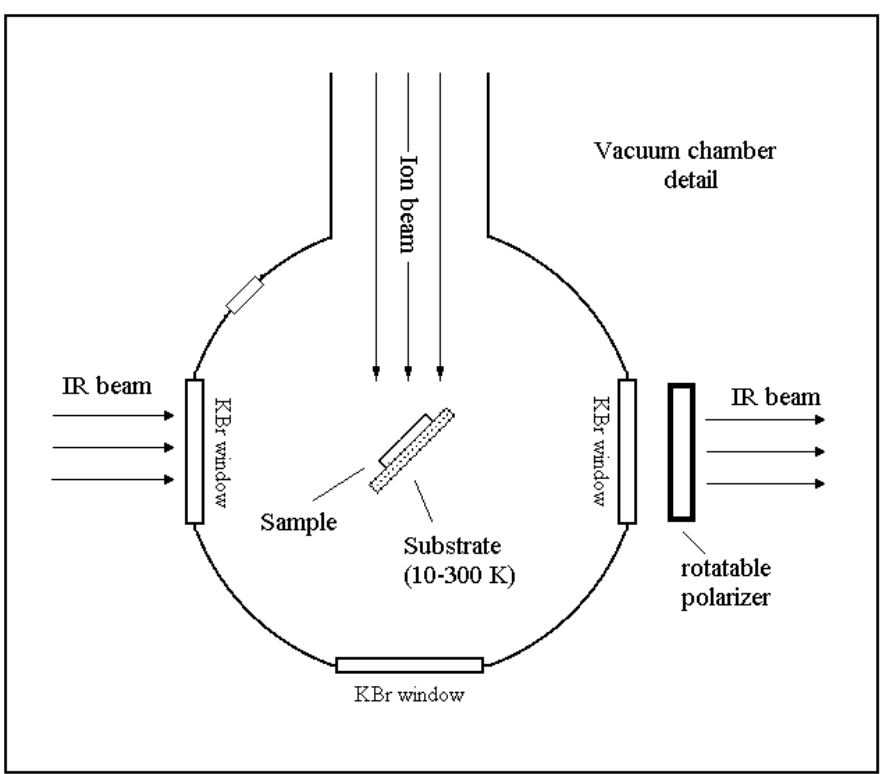

Fig. 2. Schematic top view of the vacuum chamber used for the ion irradiation of the ${ }^{13} \mathrm{C}$ grain/ice layer with $200 \mathrm{keV} \mathrm{H}^{+}$at the INAFOsservatorio Astrofisico di Catania.

fullerene-like ${ }^{13} \mathrm{C}$ film is placed in thermal contact with a closedcycle helium cryostat, whose temperature was cooled down to $17 \mathrm{~K}$. Water or oxygen was added into the chamber through a needle valve, where they froze onto the substrate. The growth of ices was monitored using an FTIR spectrometer (Bruker Vertex 70) and spectra were acquired in the $7000-400 \mathrm{~cm}^{-1}$ range at a resolution of $1 \mathrm{~cm}^{-1}$. The ice thickness was derived by the laser interference method (e.g. Fulvio et al. 2009) depositing the same amount of $\mathrm{H}_{2} \mathrm{O}$ or $\mathrm{O}_{2}$ on a clean $\mathrm{Si}$ substrate. It turned out that the thickness of the $\mathrm{H}_{2} \mathrm{O}$ film was about $250 \mathrm{~nm}$ (corresponding to 830 monolayers (ML)) and the thickness of the $\mathrm{O}_{2}$ film was about $130 \mathrm{~nm}$ (around $400 \mathrm{ML}$ ). The vacuum chamber is connected to an ion implanter ( $200 \mathrm{kV}$; Danfysik) from which ions of energy up to $200 \mathrm{keV}$ (400 keV for double ionizations) can be obtained. The ion beam produces a spot on the target larger than the area probed by the IR beam. Current densities ranged from $100 \mathrm{nA} \mathrm{cm}^{-2}$ up to a few $\mu \mathrm{A} \mathrm{cm}^{-2}$. In the setup, IR spectra can be obtained before and after ion irradiation without tilting the sample. Spectra were acquired after irradiation at low temperature. The optical depth was calculated from the transmission by using the OPUS 86 software. This enabled us to monitor further changes in profile of the $\mathrm{CO}_{2}$ bands upon further irradiation with $200 \mathrm{keV}$ protons. The solid ${ }^{13} \mathrm{CO}_{2}$ has an IR absorption band at $2275 \mathrm{~cm}^{-1}(4.40 \mu \mathrm{m})$ which is shifted from the $2345 \mathrm{~cm}^{-1}(4.26 \mu \mathrm{m})$ band of solid ${ }^{12} \mathrm{CO}_{2}$. Furthermore, the absorption bands of solid ${ }^{13} \mathrm{CO}_{2}$ are shifted from those of residual gaseous ${ }^{12} \mathrm{CO}_{2}$ present in the external path of the infrared beam, a perturbation that is present only when the quality of the dry air purge is not optimum (Raut et al. 2012).

In this series of experiments listed in Table 1 , three different carbon samples were used for the deposition of ice layers and ion bombardment with $200 \mathrm{keV}$ protons at various fluences. In experiment No. 1 , a ${ }^{12} \mathrm{C}$ sample was covered by an $\mathrm{H}_{2} \mathrm{O}$ ice layer only one time and subjected to irradiation with $200 \mathrm{keV} \mathrm{H}^{+}$up to the maximum fluence of $7.8 \times 10^{15}$ ions $/ \mathrm{cm}^{2}$. Similarly, in experiment No. 2, a ${ }^{13} \mathrm{C}$ sample was covered by an $\mathrm{H}_{2} \mathrm{O}$ ice layer and irradiated with $200 \mathrm{keV} \mathrm{H}^{+}$with the maximum fluence of $9 \times 10^{15}$ ions $/ \mathrm{cm}^{2}$. The carbon grain sample in experiment No. 3 from ${ }^{13} \mathrm{C}$ was used for multiple $\mathrm{O}_{2}$ and $\mathrm{H}_{2} \mathrm{O}$ depositions along
Table 1. Ion bombardment experiments with $200 \mathrm{keV} \mathrm{H}{ }^{+}$ions performed at INAF, Catania.

\begin{tabular}{cccc}
\hline \hline $\begin{array}{c}\text { Experiment } \\
\text { No. }\end{array}$ & Type of C-sample & Ices & $\begin{array}{c}\text { Max. fluence } \\
\text { ions cm }{ }^{-2}\end{array}$ \\
\hline 1 & ${ }^{12} \mathrm{C}$ & $\mathrm{H}_{2} \mathrm{O}$ & $7.8 \times 10^{15}$ \\
2 & ${ }^{13} \mathrm{C}$ & $\mathrm{H}_{2} \mathrm{O}$ & $9 \times 10^{15}$ \\
$3 \mathrm{a}$ & ${ }^{13} \mathrm{C}$ & $\mathrm{O}_{2}$ & $6.57 \times 10^{15}$ \\
$3 \mathrm{~b}$ & ${ }^{13} \mathrm{C}$ & $\mathrm{O}_{2}$ & $1.65 \times 10^{16}$ \\
$3 \mathrm{c}$ & ${ }^{13} \mathrm{C}$ & $\mathrm{O}_{2}$ & $1.65 \times 10^{16}$ \\
$3 \mathrm{~d}$ & ${ }^{13} \mathrm{C}$ & $\mathrm{H}_{2} \mathrm{O}$ & $1.15 \times 10^{16}$ \\
$3 \mathrm{e}$ & ${ }^{13} \mathrm{C}$ & $\mathrm{O}_{2}$ & $1.65 \times 10^{16}$ \\
\hline
\end{tabular}

with proton bombardment at maximum fluences of $6.57 \times 10^{15}$, $1.65 \times 10^{16}, 1.65 \times 10^{16}, 1.15 \times 10^{16}$, and $1.65 \times 10^{16} \mathrm{ions} / \mathrm{cm}^{2}$. This corresponds to the total fluence of $6.76 \times 10^{16} \mathrm{ions} / \mathrm{cm}^{2}$. After each individual bombardment experiment, the ice layer was removed by annealing to room temperature, and a new ice layer was deposited on the bare grains for the following experiment. In all our experiments, the penetration depth of impinging ions is greater than the thickness of the ice layer. According to SRIM (Stopping and Range of Ions in Matter) calculations, the penetration depth of $200 \mathrm{keV}$ protons impinging at $45^{\circ}$ is about $1 \mu \mathrm{m}$ for both cases of $250 \mathrm{~nm} \mathrm{H}_{2} \mathrm{O}$ ice layer and $130 \mathrm{~nm} \mathrm{O}_{2}$ ice layer on top of an amorphous carbon layer with a density of $1.7 \mathrm{~g} / \mathrm{cm}^{3}$ (Ziegler et al. 2010).

\subsection{Ion bombardment of carbon grains without ice layer}

Bare carbon grains were bombarded with $200 \mathrm{keV}$ protons. The aim of these experiments were to compare and monitor the structural changes of carbon surfaces covered by ice with those having no ice layers under ion irradiation. For this reason, fullerenelike particulate carbon layers produced from both ${ }^{12} \mathrm{C}$ and ${ }^{13} \mathrm{C}$ were prepared by the laser ablation technique as already mentioned in Sect. 2.1. The carbon layers were deposited on silicon substrates. To compare the results of these experiments with the ones performed in Catania, we applied $200 \mathrm{keV} \mathrm{H}^{+}$ions and a maximum fluence of $7 \times 10^{16}$ ions $/ \mathrm{cm}^{2}$. The sample was cooled down to $16 \mathrm{~K}$ during the experiments.

\subsection{Analytical characterization}

Because of the very complex structure of carbon materials, they have to be analyzed by different methods. In order to characterize as-produced and processed carbon materials, ambitious analytical methods such as high-resolution transmission electron microscopy (HRTEM) and field emission scanning electron microscopy (FESEM) were used. In addition, Fourier-transform infrared (FTIR) spectroscopy was performed in transmission in the wavelength range between 400 and $4000 \mathrm{~cm}^{-1}$. HRTEM gives us a direct view inside the grains and unveils the information on the structure, sizes, and shapes of the grains. With this method, one can distinguish between ordered and amorphous structures, or between plane and bent graphene layers. A JEOL JEM-3010 TEM microscope equipped with $\mathrm{L} \mathrm{LaB}_{6}$ cathode operating at $300 \mathrm{kV}$ and a point resolution of $0.21 \mathrm{~nm}$ was used. HRTEM images were recorded digitally with a CCD camera and analyzed by the Gatan Digital Micrograph 3.9.0 software. FESEM is performed with a field-emission SEM (Zeiss (LEO) 1530 Gemini). The resolution of the microscope depends on the material but it can be $2 \mathrm{~nm}$ in maximum. This special technique was used to provide information on the morphology of the condensed layers. 
Table 2. List of molecules observed after irradiation with $200 \mathrm{keV} \mathrm{\textrm {H } ^ { + }}$ ions in different ices together with their band positions and strengths used to evaluate their column densities.

\begin{tabular}{cccc}
\hline \hline Species & $\begin{array}{c}\text { Peak positions }(v) \\
\mathrm{cm}^{-1}\end{array}$ & $\begin{array}{c}\text { Band strength }(A) \\
\text { cm molecule }\end{array}$ & References \\
\hline${ }^{13} \mathrm{CO}_{2}$ & 2277 & $7.6 \times 10^{-17}$ & Yamada \& Person (1964) \\
${ }^{12} \mathrm{CO}_{2}$ & 2340 & $7.6 \times 10^{-17}$ & Yamada \& Person (1964) \\
${ }^{13} \mathrm{CO}$ & 2092 & $1.1 \times 10^{-17}$ & Loeffler et al. (2005) \\
${ }^{12} \mathrm{CO}$ & 2140 & $1.1 \times 10^{-17}$ & Jiang et al. (1975) \\
$\mathrm{H}_{2} \mathrm{O}$ & 3300 & $2 \times 10^{-16}$ & Hudgins et al. (1993) \\
$\mathrm{O}_{3}$ & 1038 & $1.4 \times 10^{-17}$ & Smith et al. (1985) \\
\hline
\end{tabular}

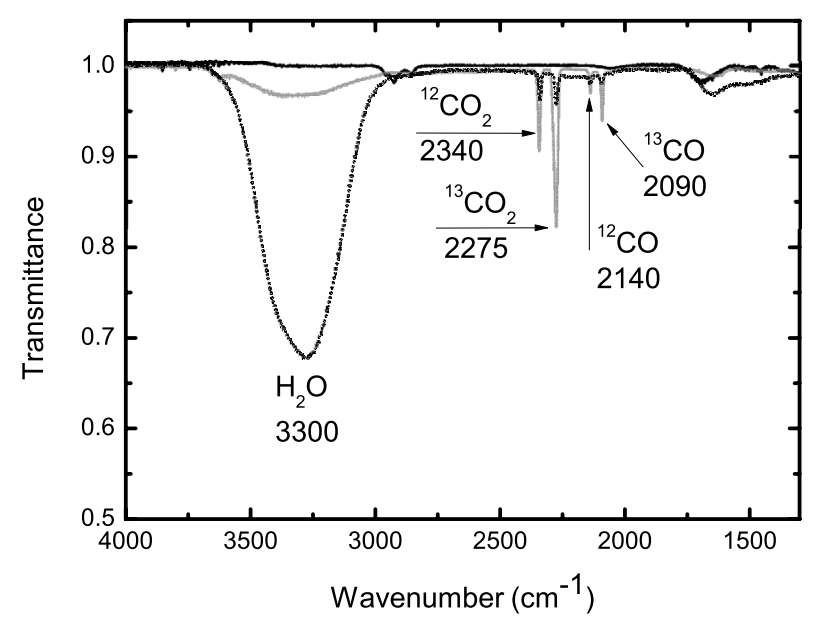

Fig. 3. IR spectra of amorphous ${ }^{13} \mathrm{C}$ (solid line), amorphous ${ }^{13} \mathrm{C}$ after $\mathrm{H}_{2} \mathrm{O}$ ice deposit (dotted line) and after $\mathrm{O}_{2}$ ice deposit (gray solid line) along with irradiation of $200 \mathrm{keV} \mathrm{H}^{+}$(experiment No. 3 in Table 1).

\section{Results}

\section{1. $\mathrm{CO}$ and $\mathrm{CO}_{2}$ formation in ices under ion bombardment}

It is already well studied that ion irradiation of simple ices produces new species both more and less volatile than those initially present in the sample. Both $\mathrm{CO}$ and $\mathrm{CO}_{2}$ can be obtained when mixtures containing simple molecules of astrophysical interest (e.g. Moore et al. 1991, 1996; Strazzulla et al. 1993; Palumbo et al. 1998) are irradiated.

In our experiments, the evolution of $\mathrm{CO}$ and $\mathrm{CO}_{2}$ in $\mathrm{H}_{2} \mathrm{O}$ and $\mathrm{O}_{2}$ ice layers bombarded with $200 \mathrm{keV}$ protons was observed. In Table 2, peak positions and band strengths of these molecules are given that are considered to evaluate their column densities. Furthermore, we analyzed the morphology and spectral properties of ${ }^{13} \mathrm{C}$ before and after processing.

The baseline-corrected IR transmission spectra of the asproduced and processed ${ }^{13} \mathrm{C}$ grains are presented in Fig. 3. OHstretching bands at around $3300 \mathrm{~cm}^{-1}$ are seen in the spectra of carbon samples covered with ices. Only weak aliphatic $\mathrm{C}-\mathrm{H}$ stretching bands between $2800-3000 \mathrm{~cm}^{-1}(3.4 \mu \mathrm{m})$ have been detected in the spectrum. In addition, weak features at 1430 and $1375 \mathrm{~cm}^{-1}$ are assigned to aliphatic $\mathrm{CH}$-deformation vibrations. But small features between 1605 and $1610 \mathrm{~cm}^{-1}$, observed in all samples, confirm the existence of aromatic $-\mathrm{C}=\mathrm{C}$ - bonds in the carbon structure. The hydrogen is mainly incorporated as saturated $-\mathrm{CH}_{2}$ or $-\mathrm{CH}_{3}$ groups probably acting as bridges between bent aromatic subunits. For more details on the fullerene-like structure and IR properties of the original condensate, we refer to the paper published by Jäger et al. (2008). In addition, narrow features of ${ }^{12} \mathrm{CO},{ }^{13} \mathrm{CO},{ }^{12} \mathrm{CO}_{2}$, and ${ }^{13} \mathrm{CO}_{2}$ molecules are distinguished in the spectrum. There is a clear shift that is about $50 \mathrm{~cm}^{-1}$ for $\mathrm{CO}$ and $65 \mathrm{~cm}^{-1}$ for $\mathrm{CO}_{2}$. Only the ${ }^{13} \mathrm{CO}$ and ${ }^{13} \mathrm{CO}_{2}$ molecules are formed from carbon atoms of the particulate material. The decrease in the intensity of aliphatic $\mathrm{C}-\mathrm{H}$ stretching modes is related to the abstraction of hydrogen from the solid carbon structures. The decreasing intensity of the water absorption band at $3300 \mathrm{~cm}^{-1}$ in the spectra of the $\mathrm{H}_{2} \mathrm{O}$ ice-covered carbon grains is accompanied by the appearance of $\mathrm{CO}$ and $\mathrm{CO}_{2}$ stretching bands, which develop during irradiation. Besides this, the $\mathrm{C} \equiv \mathrm{C}$ stretching band at $2110 \mathrm{~cm}^{-1}$ slightly increases by proton irradiation. The latter band is related to processing of carbon grains.

The column densities of $\mathrm{CO}$ and $\mathrm{CO}_{2}$ for common ${ }^{12} \mathrm{C}$ and ${ }^{13} \mathrm{C}$ at different ion fluences were calculated. To estimate the abundance of solid molecules in both laboratory samples and along the line of sight to molecular clouds, it is essential to convert the transmission spectra $I_{f}$ into optical depth units $\tau(v)=\ln \left(I_{0} / I_{f}\right)$, where $I_{0}$ is the intensity of the incident radiation (Ioppolo et al. 2009). After the baseline correction by a linear fit, the band area is calculated and finally divided by the band strength for the given molecules, listed in Table 2, to derive the column density for each considered species. We observed that by increasing the fluence of ions, the amount of $\mathrm{CO}$ and $\mathrm{CO}_{2}$ increases. Only the ${ }^{13} \mathrm{CO}$ and ${ }^{13} \mathrm{CO}_{2}$ molecules are formed from carbon atoms of the particulate material.

To check the origin of ${ }^{12} \mathrm{CO}_{2}$ and ${ }^{12} \mathrm{CO}$ bands in our spectra, we carried out control experiments where only $\mathrm{O}_{2}$ ice was deposited on an inert $\mathrm{Si}$ substrate and irradiated at $17 \mathrm{~K}$ with $200 \mathrm{keV} \mathrm{H}^{+}$ions up to fluences of $1.6 \times 10^{16} \mathrm{ions} / \mathrm{cm}^{2} .{ }^{13} \mathrm{CO}$ and ${ }^{13} \mathrm{CO}_{2}$ were not spectroscopically detected. In addition, we observed that the column density of ${ }^{12} \mathrm{CO}$ and ${ }^{12} \mathrm{CO}_{2}$ is more than one order of magnitude lower than the column density measured when $\mathrm{O}_{2}$ is deposited on $\mathrm{C}$ grains. Consequently, a part of the ${ }^{12} \mathrm{CO}$ and ${ }^{12} \mathrm{CO}_{2}$ must have been formed in the ice layer, on top of the carbon, upon ion bombardment. From previous studies, we know that the condensed grains easily adsorb molecules on their surfaces. Therefore, we conclude that the ${ }^{12} \mathrm{CO}$ and ${ }^{12} \mathrm{CO}_{2}$ molecules exclusively result from a layer of ${ }^{12} \mathrm{C}$ carbon-bearing molecules physisorbed on the surface of the fullerene-like grains after exposure to air.

Our results clearly indicate that the carbon particles of the layer beneath the ice are partly eroded. They have contributed to the formation of $\mathrm{CO}$ and $\mathrm{CO}_{2}$ molecules in the ice layer. This is proved by the formation of ${ }^{13} \mathrm{CO}$ and ${ }^{13} \mathrm{CO}_{2}$. When $\mathrm{O}_{2}$ is the only oxygen-bearing molecule, $\mathrm{CO}_{2}$ is probably formed from $\mathrm{CO}$ via the following reactions similar to a mechanism proposed by Fulvio et al. (2012):

$$
\begin{aligned}
& \mathrm{O}_{2}+\mathrm{C} \rightarrow \mathrm{CO}+\mathrm{O} \\
& \mathrm{CO}+\mathrm{O} \rightarrow \mathrm{CO}_{2}
\end{aligned}
$$


The efficiency of $\mathrm{CO}$ and $\mathrm{CO}_{2}$ formation in an $\mathrm{H}_{2} \mathrm{O}$ ice layer is about one-fifth of its efficiency in an $\mathrm{O}_{2}$ ice layer. The oxidation process that is responsible for the formation of $\mathrm{CO}$ and $\mathrm{CO}_{2}$ is probably different to the one observed in $\mathrm{O}_{2}$ ice. An impinging ion $\mathrm{X}$ can directly break a water molecule:

$\mathrm{X}+\mathrm{H}_{2} \mathrm{O} \rightarrow \mathrm{H}+\mathrm{OH}$.

Near the carbon-water interface, $\mathrm{OH}$ radicals can react with the carbon to form $\mathrm{CO}_{2}$ in steps via creation of an intermediate $\mathrm{CO}$, since it is unlikely that two $\mathrm{OH}$ radicals are available to oxidize a single carbon atom at the same instant as explained in more detail in Raut et al. (2012):

$\mathrm{OH}+\mathrm{C}(\mathrm{s}) \rightarrow \mathrm{H}+\mathrm{CO}$

$\mathrm{OH}+\mathrm{CO} \rightarrow \mathrm{H}+\mathrm{CO}_{2}$.

\subsection{Estimation of missing $C$ after ion bombardment}

The bombardment experiments have clearly proven that the formation of ${ }^{13} \mathrm{CO}$ and ${ }^{13} \mathrm{CO}_{2}$ was at the expense of solid carbon grains. We, therefore, estimated the amount of carbon removed from the carbon particles by the formation of $\mathrm{CO}$ and $\mathrm{CO}_{2}$ in the experiments No. 3a-e. Based on the column density of ${ }^{13} \mathrm{CO}$ and ${ }^{13} \mathrm{CO}_{2}$ plotted as a function of the fluence, the formation rate of ${ }^{13} \mathrm{CO}$ and ${ }^{13} \mathrm{CO}_{2}$ in this experiment was calculated. The experimental data were fitted by an exponential curve $y=A\left(1-\mathrm{e}^{-\sigma \Phi}\right)$, where $y$ is the ${ }^{13} \mathrm{CO}$ or ${ }^{13} \mathrm{CO}_{2}$ column density (molecules $/ \mathrm{cm}^{2}$ ), $A$ the asymptotic value (molecules $/ \mathrm{cm}^{2}$ ), $\sigma$ the total cross section $\left(\mathrm{cm}^{2} /\right.$ ion), and $\Phi$ the proton fluence (ions $\left./ \mathrm{cm}^{2}\right)$. The formation rate, $R$ (molecules/ion), was calculated by $A \times \sigma$. Figure 4 shows the column density of ${ }^{13} \mathrm{CO}$ and ${ }^{13} \mathrm{CO}_{2}$ acquired at different fluences of $200 \mathrm{keV}$ protons upon irradiating an $\mathrm{O}_{2}$ ice layer deposited on a ${ }^{13} \mathrm{C}$ grain layer and the corresponding fit curve.

Summing up the data of all five irradiation experiments $(3 \mathrm{a}-$ e), we obtained a total number of $5.09 \times 10^{17}$ carbon atoms $/ \mathrm{cm}^{2}$ converted from the solid carbon into ${ }^{13} \mathrm{CO}$ and ${ }^{13} \mathrm{CO}_{2}$ molecules. This is calculated from the formation rate of ${ }^{13} \mathrm{CO}$ and ${ }^{13} \mathrm{CO}_{2}$ multiplied by the total fluence $6.76 \times 10^{16}$ ions $/ \mathrm{cm}^{2}$.

The amount of $\mathrm{C}$ removed from the particulate carbon layer was calculated by the formation rate, i.e. 4.7 molecules/ion for ${ }^{13} \mathrm{CO}_{2}$ and 2.9 molecules/ion for ${ }^{13} \mathrm{CO}$, multiplied by the number of ions calculated from the total fluence $6.76 \times 10^{16}$ ions $/ \mathrm{cm}^{2}$ multiplied by the area of the irradiated surface, which gives $5.32 \times 10^{16}$ ions. Finally, we obtained a total number of removed carbon molecules or atoms of $4 \times 10^{17}$ molecules.

Based on the volume of the carbon layer that was irradiated, we determined the number of originally available carbon atoms. The fraction of atoms that was transferred into molecules from the solid corresponds to about $50 \%$. In our study, we removed a carbon layer of about $60 \mathrm{~nm}$ from the original carbon film with $5.32 \times 10^{16}$ ions corresponding to $1.13 \times 10^{-15} \mathrm{~nm} /$ ion erosion rate. The reduction of the carbon layer thickness by about $50 \%$ is also visible in the FESEM image that shows the topography of the irradiated and nonirradiated parts of the sample (Fig. 5).

\subsection{Structural modifications of the carbonaceous material under ion bombardment}

The HRTEM provides information on the level of ordering in the primary particles by imaging graphene subunits or lattice fringes of graphite-like structures. It is already known that the possibilities for carbon forming solid structures are extremely manifold. Tetrahedrally coordinated carbon atoms in the $\mathrm{sp}^{3}$ hybridization

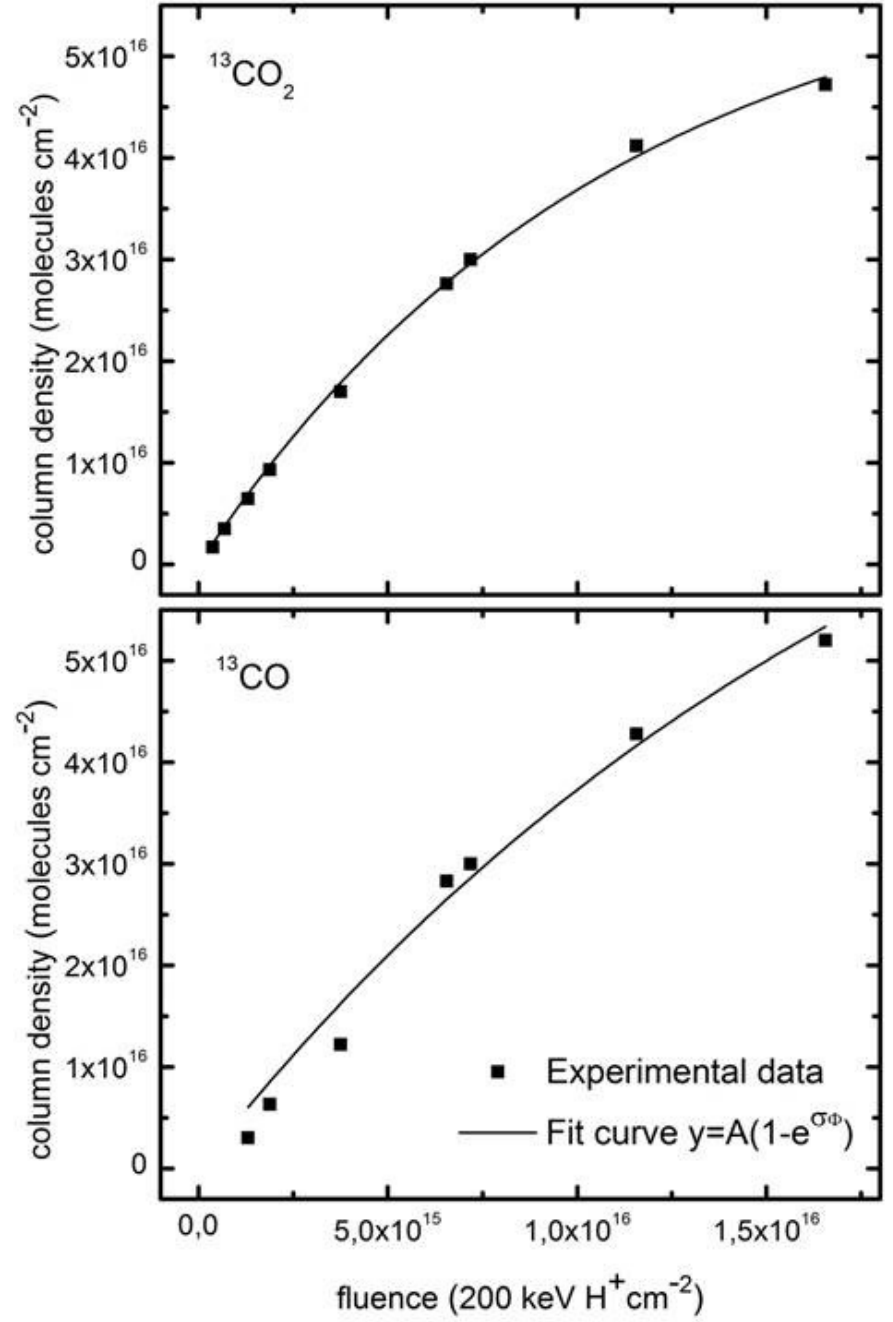

Fig. 4. Column densities of ${ }^{13} \mathrm{CO}$ (lower graph) and ${ }^{13} \mathrm{CO}_{2}$ (upper graph) versus the fluence. The formation rates of both molecules were derived from the fit of the experimental data with a standard deviation of $\delta R= \pm 0.38$ (molecules/ion).

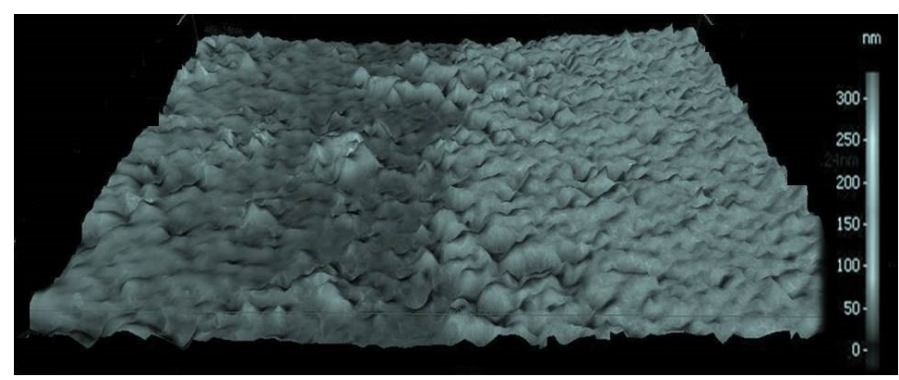

Fig. 5. Topographic view produced from a FESEM micrograph showing the erosion of the carbon layer after ion bombardment with $6.76 \times$ $10^{16}$ protons $/ \mathrm{cm}^{2}$. The left part is the bombarded area whereas the right and bright surface represents the nonirradiated part of the sample. The scale on the right side corresponds to the layer thickness.

state can build up either diamond or diamond-like amorphous structures, whereas $\mathrm{sp}^{2}$ hybridized carbon atoms form planar structures (graphite or graphite-like). Apart from these two distinct hybridization states, mixed hybridization characterized by curved structures is possible (fullerenes or fullerene-like structures). In a carbon grain, all kinds of hybridization states (sp, $\mathrm{sp}^{2}, \mathrm{sp}^{3}$, and curved graphene layers) may occur together. 

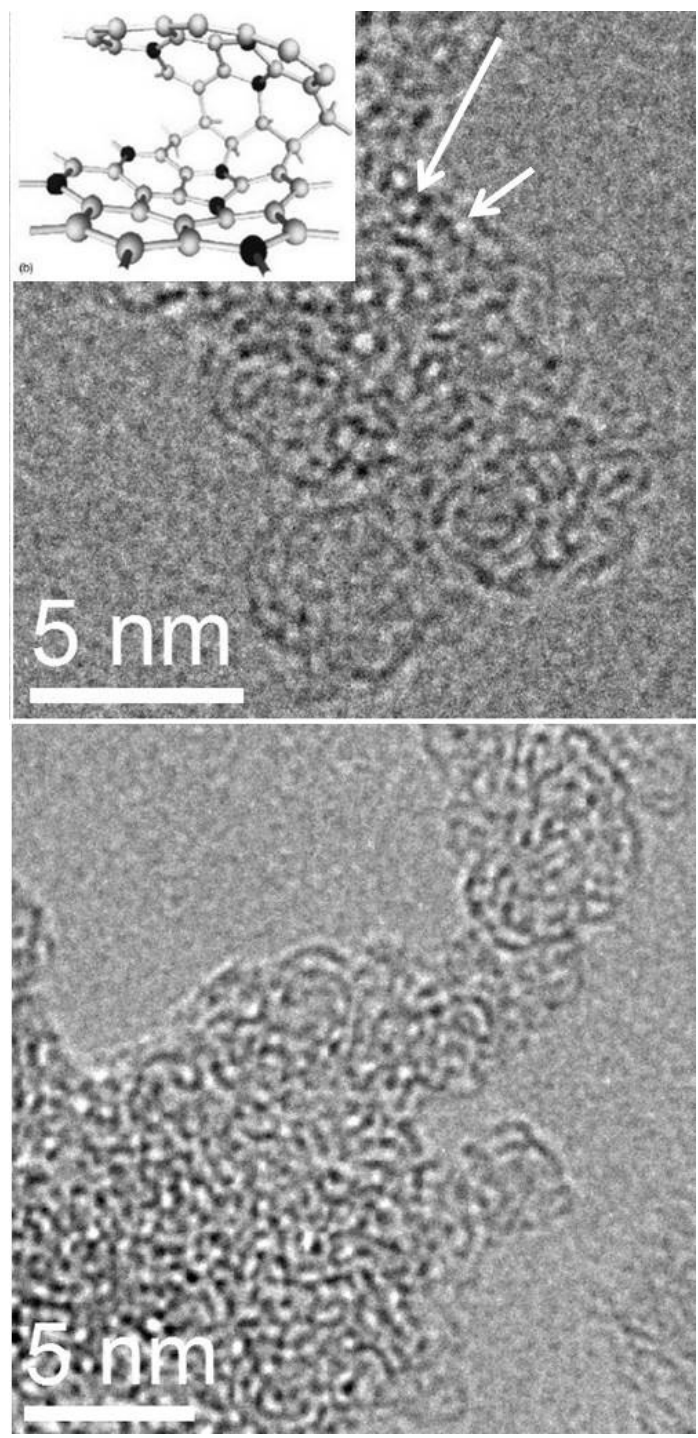

Fig. 6. HRTEM image of fullerene-like carbon grains before ion bombardment. The inset demonstrates the cross-linking between the bent graphene layers. The arrows mark individual fullerenes between the grains.

The gas-phase condensed grains used in this study are built of amorphous, fullerene-like structures, the same as those obtained by laser ablation of graphite in quenching gas atmospheres (Jäger et al. 2008). The comparison of ${ }^{12} \mathrm{C}$ and ${ }^{13} \mathrm{C}$ particulate carbon layers does not show any structural difference in the condensates. Figure 6 presents images of fullerene-like carbon before ion irradiation. The fullerene-like structure arises from the presence of bent and cross-linked graphene planes. The very fluffy agglomerates are dominated by chain-like arrangements of particles. Finally, a very porous carbon layer composed of small fullerene-like grains was observed in the micrographs. Individual grains were found to range between 1 and $3 \mathrm{~nm}$. In addition, fullerenes of different sizes are observed in the condensate.

The ${ }^{13} \mathrm{C}$ particulate sample produced by laser ablation was used for five experiments (3a-e) comprising ice deposition with $\mathrm{O}_{2}$ or $\mathrm{H}_{2} \mathrm{O}$ and subsequent ion bombardment of the grain/ice layer after each deposition. Therefore, the carbon layer (sample No. 3) should show the utmost changes in structure and morphology. Ion-irradiation of $\mathrm{O}_{2}$ or $\mathrm{H}_{2} \mathrm{O}$ ice on carbonaceous dust
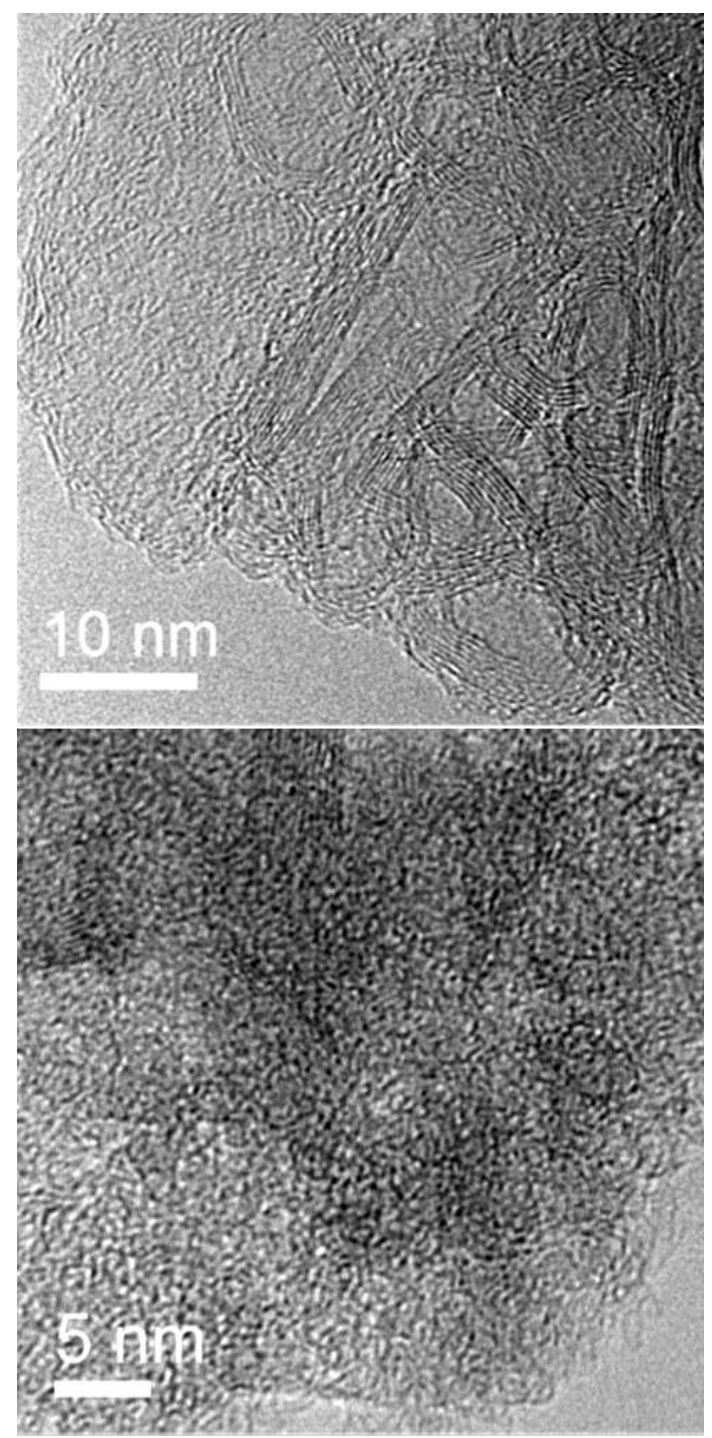

Fig. 7. HRTEM images of the carbon structures after ice deposition and irradiation-induced processing (sample $3 \mathrm{e}$ in Table 1). The upper image shows a strongly processed area of carbon material representing graphitic structures. The lower image displays material that is less processed compared to the top layer. It still shows a similarity to the original fullerene-like material but with longer graphene layers.

grain surfaces that act as a reservoir of carbon triggers reactions between the carbon and the ice at the interface. $\mathrm{C}-\mathrm{C}$ and $\mathrm{C}-\mathrm{H}$ bonds are dissociated by the energetic ions, and finally, $\mathrm{CO}$ and $\mathrm{CO}_{2}$ molecules are formed. Besides the formation of molecules, structural rearrangements in the remaining carbon can be observed. Figure 7 shows an HRTEM image of ${ }^{13} \mathrm{C}$ grains after proton bombardment. The upper image provides insight into a strongly processed area of carbon material observed at the interface between grains and ice. Fibers showing the typical lattice fringes of graphitic structures $\left(d_{002}=0.365 \mathrm{~nm}\right)$ can be observed. The graphitic material was clearly produced from the original fullerene-like material upon the proton bombardment.

The FESEM images (Fig. 8) confirm that graphitization occurred in a surface layer after the intensive bombardment. This surface layer can be clearly distinguished from the grain-like structure underneath. In addition, the growth of the grains and densification of agglomerates are visible. The thickness of the graphitic layer was estimated to be in the range of 30 to $50 \mathrm{~nm}$. 


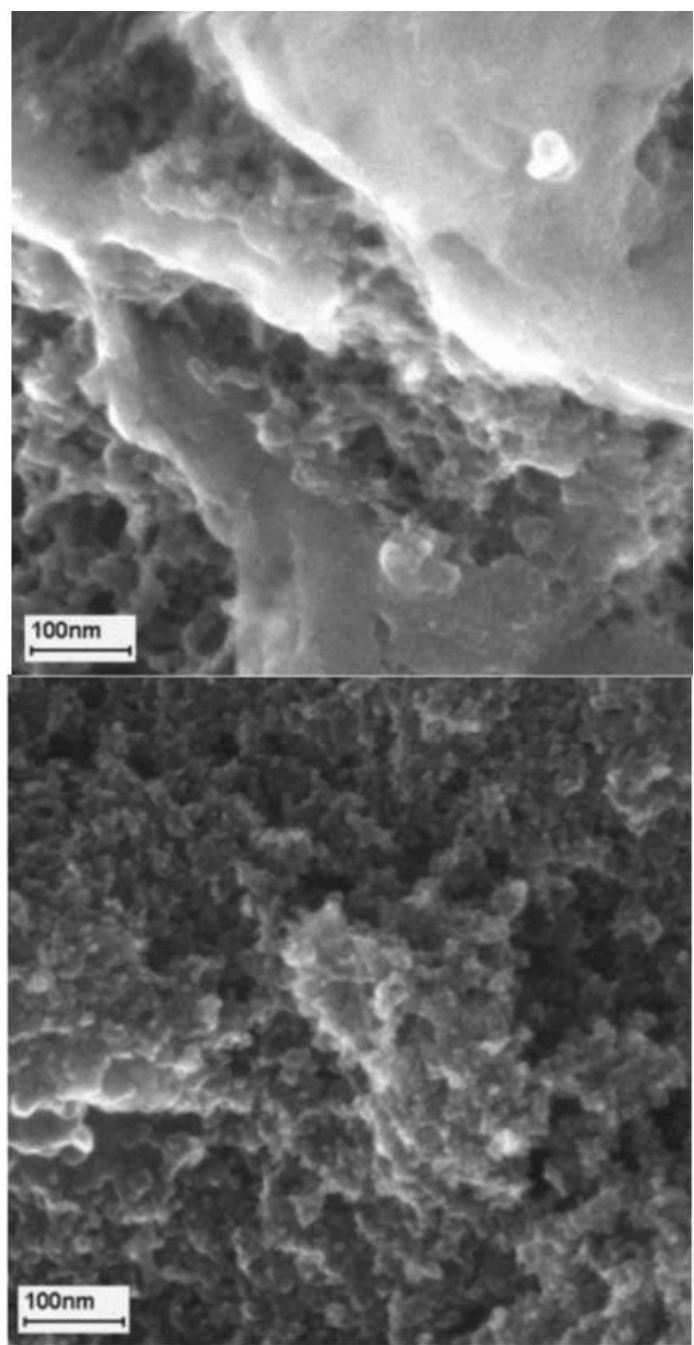

Fig. 8. FESEM image of carbon grains before (lower image) and after $200 \mathrm{keV}$ proton bombardment with $6.76 \times 10^{16}$ ions $/ \mathrm{cm}^{2}$ (top image).

To interpret the results of structural modifications by proton bombardment, the interaction of protons with the target material has to be understood. The penetration depth of $200 \mathrm{keV}$ protons into a very porous layer of carbon grains, covered by either 130 or $250 \mathrm{~nm}$ of $\mathrm{O}_{2}$ or $\mathrm{H}_{2} \mathrm{O}$ ice, respectively, is difficult to determine by SRIM calculations. Assuming a dense, non-porous carbon layer, the thickness measured by means of a quartz micro balance, was about $120 \mathrm{~nm}$. SRIM simulations, which take a non-porous carbon layer with the density of $2.2 \mathrm{~g} / \mathrm{cm}^{3}$ beneath the ice into account, calculated penetration depths between 220 and $330 \mathrm{~nm}$. The protons are finally stopped in the $\mathrm{KBr}$ substrate. However, the chain-like, porous structure of the grain agglomerates is much less dense, resulting in a real layer thickness ranging between 900 and $1100 \mathrm{~nm}$ and corresponding to a density of around $0.35 \mathrm{~g} / \mathrm{cm}^{3}$. During their trajectory, they lose energy and deposit this energy in the target mainly in the form of electronic energy. Consequently, ionization and bond breaking, as well as annealing processes, are triggered in the material. These processes are responsible for the structural modification of the fullerene-like grains. However, a gradual modification of the carbon material is observed from the surface to the bottom of the carbon layer. Beneath the strongly graphitized upper layer, we observe less processed carbonaceous material characterized by longer graphene layers than in the original material, and distinct grain structures. The different levels of carbon grain processing are shown in Fig. 9. In addition, the formation of more closed buckyonions and finally less modified grains with abundant fullerenes are observed. The energetic protons cause the abstraction of $\mathrm{H}$ from aliphatic $-\mathrm{C}-\mathrm{H}$ bonds in the periphery of fullerene-like aromatic structures. Infrared analysis recorded during the irradiation experiments confirmed the destruction of $\mathrm{C}-\mathrm{H}$ bonds under irradiation. Consequently, reactions between dangling bonds at the periphery of fullerene fragments can lead to the formation of fullerenes and bucky onions of different sizes. This scenario corresponds well to the results seen in the HRTEM analysis.

To understand the processing of carbonaceous materials upon ion bombardment, bare carbonaceous material (without ice layer) was studied. It should be noted that the fluence of protons was comparable to the total proton fluence used for the ice-covered sample 3 (a-e). Figure 10 presents the HRTEM analysis of the processed carbonaceous grains. Generally, an identical gradual processing of the carbon material as shown in Figs. 8 and 9 could be detected. On the surface, the formation of graphitic structures was observed. However, this graphitic layer was smaller compared to those observed in the ice-covered sample. A possible explanation could be that the dissociation of bonds and formation of atoms in the ice layer may contribute to the graphitization process at the interface ice/grains. They can additionally react with $\mathrm{C}-\mathrm{C}$ and $\mathrm{C}=\mathrm{C}$ bonds in the carbon grains and therefore increase the rate of bond dissociation in the carbon layer upon ion bombardment.

\section{Astrophysical applications}

\subsection{Implication for molecular formation and erosion of solid carbon for molecular clouds and protoplanetary disks}

Interactions between ice and grains are expected in the ISM, such as in dense molecular clouds, in clouds with embedded protostars, or in protoplanetary disks, where dust grains coated with ice are continually bombarded by $\mathrm{GeV}$ cosmic rays or stellar winds.

Solid $\mathrm{CO}$ and $\mathrm{CO}_{2}$ are the foremost molecules formed at low temperature after processing of $\mathrm{C}$ - and O-bearing ices. In the cold ISM, the formation of observed $\mathrm{CO}_{2}$ could be due to oxidation of the abundant solid CO. In this process, oxygen atoms may plate out onto grains in dense clouds or result from the dissociation of adjacent molecules in the ice, such as $\mathrm{O}_{2}$ or $\mathrm{H}_{2} \mathrm{O}$ by Ly $\alpha$ photons, cosmic rays, or stellar wind ions. Raut \& Baragiola (2011) find that the reaction of $\mathrm{CO}$ and cold $\mathrm{O}$ atoms is relatively inefficient at making $\mathrm{CO}_{2}$, mostly because oxygen reacts primarily with $\mathrm{O}$ atoms to form $\mathrm{O}_{2}$ and $\mathrm{O}_{3}$. The presence of $\mathrm{CO}$ ice in grain mantles is generally explained in terms of direct condensation of $\mathrm{CO}$ molecules from the gas phase. On the other hand, it is thought that $\mathrm{CO}_{2}$ forms in the solid phase through grain surface reactions and energetic processing of ice mantles. Former laboratory results (Ioppolo et al. 2009, 2013) not only account quantitatively for the column density of observed interstellar $\mathrm{CO}_{2}$ but also provided a good spectroscopic analog of the interstellar features supporting the hypothesis that interstellar solid $\mathrm{CO}_{2}$ is formed after ion irradiation and UV photolysis of icy mantles. This, however, does not exclude the possibility that other formation routes, such as grain surface reactions, contribute to the production of the observed interstellar solid $\mathrm{CO}_{2}$.

Our experiments have shown that $\mathrm{CO}$ and $\mathrm{CO}_{2}$ are formed from the solid phase. That means that solid carbon is eroded not only by supernova shock waves but also by 

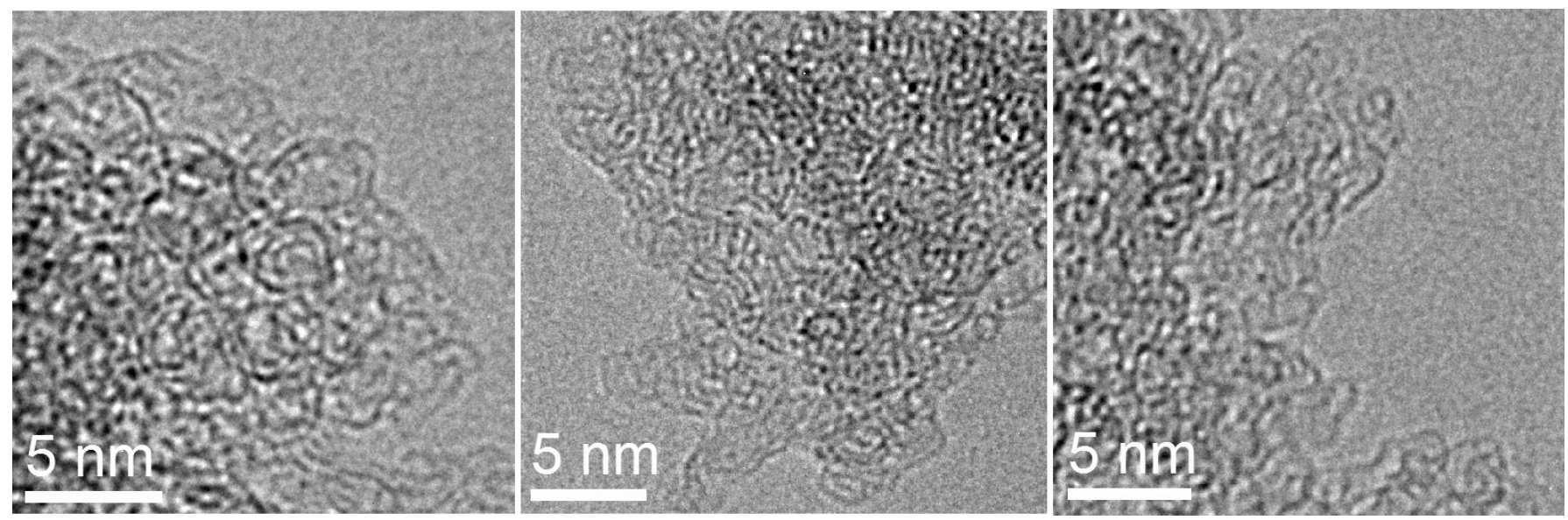

Fig. 9. Series of images presenting material with different levels of proton-induced processing. The three types of carbon structures were observed underneath the graphitic layer after complete bombardment of sample 3 with the total fluence of $6.76 \times 10^{16}$ ions $/ \mathrm{cm}^{2}$. The particles are still visible, but they contain longer graphene layers compared to those observed in the original grains. In addition, one can see the formation of more closed buckyonions and fullerenes. The right micrograph finally shows grains, which are still similar to the unprocessed fullerene-like grains.
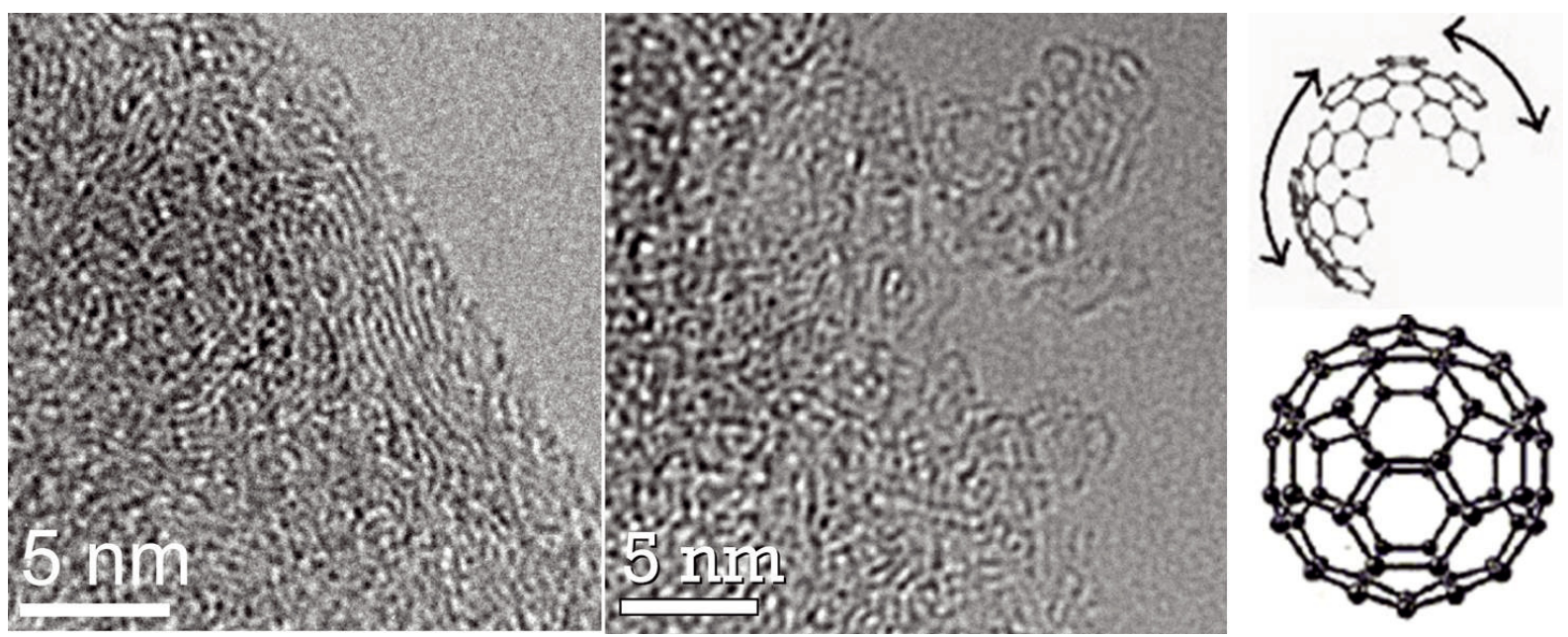

Fig. 10. Graphitic structures observed after bombardment of bare carbonaceous grains with $200 \mathrm{keV}$ protons at total fluence of $6.76 \times 10^{16}$ ions $/ \mathrm{cm}^{2}$ $(l e f t)$. Fullerenes and bucky onions (middle), detected beneath the graphite layer, demonstrate the conversion of curved fullerene fragments into more closed structures (right).

ion-induced molecule formation in ices deposited on carbon grains. Assuming that ice deposition and simultaneous ion irradiation may occur repeatedly, such processes may drastically reduce the amount of solid carbon in the ISM and in other astrophysical environments.

The erosion of carbon grains due to $\mathrm{CO}$ and $\mathrm{CO}_{2}$ formation at the interface between carbonaceous grains and ice layers is not well known yet. Our experiments have provided quantitative results on the level of carbon-grain erosion. A total irradiation fluence of $6.76 \times 10^{16} \mathrm{H}^{+} \mathrm{cm}^{-2}$ applied to ice-covered carbon grains has resulted in the erosion of about $50 \%$ of the solid material here considered. The applied fluence is equivalent to the proton bombardment during the grains' lifetime of about $10^{8}$ years in dense molecular clouds. Based on the assumption of an effective monoenergetic ion irradiation between 1 and $9 \mathrm{MeV}$ protons $\mathrm{cm}^{-2} \mathrm{~s}^{-1}$, an astrophysically relevant fluence of $3.2 \times 10^{15}$ to $2.9 \times 10^{16}$ protons $\mathrm{cm}^{-2}$ during the lifetime of a grain can be calculated (Mennella et al. 2004). The thickness of the ice layers used in our study is much smaller than expected for ice layers in dense molecular clouds. Based on experimental studies performed by Lamberts et al. (2014), ice layers of more than a few thousand monolayers for water ice are realistic. As a result, the erosion efficiency provided in this paper represents an astrophysically relevant quantity.

The results presented in this paper confirm previous findings that $\mathrm{CO}$ and $\mathrm{CO}_{2}$ are formed after ion bombardment and UV photolysis of C-grains covered by water and oxygen ice (Mennella et al. 2004, 2006; Gomis \& Strazzulla 2005; Fulvio et al. 2012; Raut et al. 2012). Similarly, in our experiments with $\mathrm{H}_{2} \mathrm{O}$ and $\mathrm{O}_{2}$ ice deposits, the intensity of the aliphatic $\mathrm{C}-\mathrm{H}$ stretching modes of the $\mathrm{CH}_{2}$ and $\mathrm{CH}_{3}$ functional groups at 2950, 2925 , and $2860 \mathrm{~cm}^{-1}$ decreases. The same trend is observed for the associated $\mathrm{C}-\mathrm{H}$ bending modes at 1457 and $1378 \mathrm{~cm}^{-1}$.

The surface chemistry model predicts that $\mathrm{CO}$ molecules, which are present in the grain mantle, react with accreting atomic oxygen to form $\mathrm{CO}_{2}$. In the case of energetic processing of ice mantles, carbon dioxide forms by reaction of C-bearing molecules (primarily $\mathrm{CO}$ ) with $\mathrm{O}$ atoms, $\mathrm{OH}$ radicals and coming from the dissociation of other molecules present in the mantles (mainly $\mathrm{H}_{2} \mathrm{O}$ and $\mathrm{O}_{2}$ in the polar and nonpolar phases, respectively). A common aspect of these formation schemes is the presence of $\mathrm{C}$ atoms in $\mathrm{C}$-bearing species (mainly $\mathrm{CO}$ ) in the ice mantles. However, the performed study reveals the formation of $\mathrm{CO}$ from reactions between solid carbon and oxygen-bearing ice 
at the interface. This has been explicitly demonstrated by the detection of ${ }^{13} \mathrm{CO}$ and ${ }^{13} \mathrm{CO}_{2}$. The $\mathrm{CO}$ turns into $\mathrm{CO}_{2}$ by further oxidation. The findings should have strong influence on existing chemical models for the formation of $\mathrm{CO}$ and $\mathrm{CO}_{2}$.

Lee et al. (2010) have shown that the bulk composition of the Earth is very carbon poor compared to the material available at formation. The deficiencies of solid carbon extends into the asteroid belt. It implies that carbon is already missing in the initial steps of planet formation including the growth of submicron silicate and carbon grains in the solar nebula. The authors have discussed a mechanism wherein pristine carbon grains are primarily destroyed by oxygen atoms. Our experimental results on ion-induced erosion of solid carbon provide another mechanism for the destruction of carbonaceous solids and the abundant formation of $\mathrm{CO}$ and $\mathrm{CO}_{2}$. The formed $\mathrm{CO}$ and possibly $\mathrm{CO}_{2}$ could be the origin of more complex organic materials finally incorporated into planetesimals and cometesimals (Bergin et al. 2014). This represents an additional source for organic carbonaceous material in meteorites and comets.

\subsection{The survival of carbon dust on the surface of icy objects in the solar system}

The surfaces of most of the satellites of Jupiter, Saturn, and Uranus are dominated by the presence of water ice, with traces of other species among which are carbon dioxide $\left(\mathrm{CO}_{2}\right)$, and non-ice species that include sulfur and carbon-bearing solid materials (for a recent review see Clark et al. 2013). Carbon dioxide has been detected on the surfaces of some icy objects such as Europa, Ganymede and Callisto (McCord et al. 1997, 1998; Hibbitts et al. 2002, 2003): on the surface and in the exosphere around the Saturnian satellites Rhea and Dione (Teolis et al. 2010; Teolis \& Waite 2012) and on Ariel, a Uranian satellite (Grundy et al. 2003).

Most of these icy objects are embedded in the magnetospheres of their respective planets and consequently are exposed to very intense fluxes of energetic ions and electrons. Energetic processing produces a number of effects such as sputtering (Baragiola 2005; Johnson et al. 2008), structural alteration (Baratta et al. 1991; Moore \& Hudson 1992; Leto \& Baratta 2003; Famá et al. 2010) and chemical processing (Moore et al. 2001; Strazzulla et al. 2001; Jones et al. 2011) that, for decades, have been simulated in the laboratory (for a recent review see Strazzulla 2011). As an example, chemical alteration leads to the formation of hydrogen peroxide $\left(\mathrm{H}_{2} \mathrm{O}_{2}\right)$ as demonstrated by many experimental results (Moore \& Hudson 2000; Gomis et al. 2004; Loeffler et al. 2006; Zheng et al. 2006) and has been found on the surface of Europa (Carlson et al. 1999), Ganymede and Callisto (Hendrix et al. 1999).

The results presented are relevant to contributing to a better understanding of the so-called "carbon cycle" in which the carbon-bearing species observed on the icy moon-carbon-based solid materials, carbonates (and carbon chains), and $\mathrm{CO}_{2}$ are continuously recycled. It has already been suggested that the observed carbon dioxide is produced by radiolytic processes induced at the interface between water ice and solid carbon (Gomis \& Strazzulla 2005), possibly delivered as carbonaceous grains from micrometeorites and interplanetary and/or interstellar dust bombardment. These results reinforce that suggestion and allow us to evaluate the "lifetime" of carbonaceous materials present on the surfaces of the icy moons. In fact, once deposited on the icy surfaces, a carbonaceous grain will be rapidly covered by the abundant water ice and irradiated by the environmental energetic ion (and electron) populations. The lifetime of a carbon layer covered by water ice is given by

$\tau=\frac{d}{3.15 \times 10^{7} \Phi Y}$

where $\tau$ (years) is the lifetime of a carbon layer of thickness $d$ (atoms $\mathrm{cm}^{-2}$ ), $\Phi$ represents the ion flux (ions $\mathrm{cm}^{-2} \mathrm{~s}^{-1}$ ), and $Y$ the total yield of $\mathrm{C}$ atoms removed per impact. The high-energy magnetospheric ion populations bombarding the surfaces of the icy moon are dominated by protons at energies of $10-1000 \mathrm{keV}$ that are perfectly simulated by the present experiments. As examples, Madey et al. (2002) report the flux of ions at the surface of Europa and Dione to be $3 \times 10^{8}$ and $10^{12} \mathrm{~cm}^{-2} \mathrm{~s}^{-1}$, respectively. Assuming that the yield of carbon atom removal is, for all of the ions, the same as measured in the present experiment, i.e. 4.7 carbon atoms/ion, we can evaluate by Eq. (1) the lifetime of a grain (or a layer) having a thickness of $10^{20}$ atoms $\mathrm{cm}^{-2}$, i.e. of about ten micrometers. The result is $\tau=2.2 \times 10^{3}$ years for a grain on the surface of Europa and $\tau=0.7$ years on Dione. These are very short times that allow the carbon atoms delivered as solid material on the surfaces to be converted in icy species (mostly $\mathrm{CO}_{2}$ and $\mathrm{CO}$ ) and become available for a further radiolytic-induced chemical (or even biological) processing. Once formed, carbon dioxide is in fact mixed with water ice (and other trace species). Energetic processes then induce the formation of carbonic acid as demonstrated by several experiments (Boduch et al. 2011; Gerakines et al. 2000; Peeters et al. 2010; Pilling et al. 2010; Zheng \& Kaiser 2007); this could be a first step in further chemical evolution particularly where additional icy species are present. Irradiated mixtures also present a "red" continuum in the UV that mimic some astronomical observations well, as recently demonstrated (Jones et al. 2014).

It is obviously relevant to evaluate the number of carbon atoms that, when delivered on the surfaces of icy satellites, is converted to $\mathrm{CO}_{2}$ first and is then available for a chemical evolution. In a recent review of the space weathering of solar system bodies, Bennett et al. (2013) report the available data on the mass flux of dust on the surfaces of several objects in the solar system (see their Table 3). While those fluxes are not known for objects in the Jupiter system, they are available for the Saturnian and Uranian systems. At Dione the flux is estimated to be $1.4 \times 10^{16} \mathrm{~g} \mathrm{~cm}^{-2} \mathrm{~s}^{-1}$ (Porter et al. 2010). Assuming that $1 \%$ of the delivered mass is made of carbon and in view of the fast transformation of solid carbon in carbon dioxide, we can estimate the total amount of carbon that is converted in $3.5 \times 10^{9}$ years (i.e. from the end of the late heavy bombardment). The result is that as much as $0.15 \mathrm{~g} \mathrm{~cm}^{-2}$ can be converted on the surface of Dione. When integrated over the surface area of the satellite $\left(4 \times 10^{16} \mathrm{~cm}^{2}\right)$, this number results in $6 \times 10^{15} \mathrm{~g}$. This is a large number that induces us to conclude that this process is extremely relevant and allows large quantities of solid carbon to be converted and participate in the chemical evolution of icy bodies.

\subsection{Consequences of ion-induced structural changes of carbonaceous dust}

Our experimental studies have demonstrated that ice-covered or bare fullerene-like carbon grains undergo strong structural modification upon $200 \mathrm{keV}$ proton bombardment. The fullerene-like carbon grains are very small and rich in defects. They are characterized by very large and reactive surfaces and therefore, the process of $\mathrm{CO}$ and $\mathrm{CO}_{2}$ formation from the carbon reservoir of the grains is very efficient. A similar reactivity is expected 
for hydrogenated amorphous carbon, but grains composed of large and plane graphene layers or of graphitic subunits should show less reactivity owing to a higher stability of the aromatic $-\mathrm{C}=\mathrm{C}-$ bonds compared to single bonds and a low content of $-\mathrm{C}-\mathrm{H}$ groups. Therefore, a less efficient $\mathrm{CO}$ and $\mathrm{CO}_{2}$ formation at the end of the lifetime of a grain and a saturation effect could be possible. However, quantitative studies of the erosion of graphitic materials are still missing. The bombardment of icy and bare carbonaceous grains irradiated with comparable fluences of $200 \mathrm{keV}$ protons show slight differences in structural modifications. The graphitization of bare grains is less efficient than icy carbon grains. Generally, the electronic energy loss of the penetrating ions causes ionization, bond breaking, and annealing in the target material. Consequently, the restructuring of carbon bonds and the final graphitization of grains can be expected. However, the different levels of graphitization between icy and bare grains have not been completely understood so far. The ice layer and the formation of $\mathrm{CO}$ and $\mathrm{CO}_{2}$ may also influence the graphitization process.

The observed structural changes have a strong influence on the spectral properties of carbonaceous material. The absorption efficiency in the IR up to the submillimeter range is higher for graphitic material than for hydrogenated amorphous or fullerene-like carbon materials and the strength of $\mathrm{C}-\mathrm{H}$ bands due to $\mathrm{C}-\mathrm{H}$ vibrations (such as $3.4,3.3,6.8$, and $7.3 \mu \mathrm{m}$ ) is decreased (Jaeger et al. 1998). The absorption efficiency $Q_{\text {abs }}$ of carbonaceous particles in the far-IR follows the power law $Q_{\mathrm{abs}} / a \sim \lambda^{-\beta}$, where a is the radius of the particles. The spectral index $\beta$ depends strongly on the internal structure of the carbon materials. Beta ascends from about one to two for spherical grains with increasing graphitization as predicted by theory. However, absorption coefficients of graphitic materials depend strongly on the morphology of the grains in the far-IR range. Calculations of the absorption efficiency of a continuous distribution of ellipsoids that simulates the agglomeration of grains show that graphitized carbon grains have a much lower index. This is caused by percolation effects, which are present in graphitized samples that contain free charge carriers. In the case of conducting materials such as graphite, an increase in the far-IR opacity and a flat absorptivity are expected for aggregated particles or deviations in the shape of particles from a sphere (Stognienko et al. 1995; Henning et al. 1995). Therefore, structural modifications of carbonaceous materials and erosion of grains have to be considered in the relevant dust models.

\section{Conclusions}

The formation of $\mathrm{CO}$ and $\mathrm{CO}_{2}$ molecules was studied upon the $200 \mathrm{keV} \mathrm{H}^{+}$bombardment of $\mathrm{O}_{2}$ and $\mathrm{H}_{2} \mathrm{O}$ ice-coated carbonaceous grains at the interface between ice and grains. The grains were produced from ${ }^{13} \mathrm{C}$ graphite targets by gas-phase condensation. We showed that $\mathrm{CO}$ and $\mathrm{CO}_{2}$ were formed, and the total number of $\mathrm{CO}$ and $\mathrm{CO}_{2}$ molecules removed from the carbon upon ion irradiation was determined to be $4 \times 10^{17}$ atoms (see Sect. 3.2). These molecules are exclusively formed from the carbon grains. We have determined the number of originally available carbon atoms based on the volume of the carbon layer that was irradiated. The fraction of atoms that are transferred into molecules from the solid corresponds to about 50\%. In our study, we have removed a carbon layer of about $60 \mathrm{~nm}$ from the original carbon film with $5.32 \times 10^{16}$ ions corresponding to $1.13 \times 10^{-15} \mathrm{~nm} /$ ion erosion rate for an oxygen ice layer.

The lifetime of a grain (or a layer) having a thickness of $10^{20}$ atoms $\mathrm{cm}^{-2}$, i.e. of about ten micrometers on icy objects of the solar system, was calculated on the basis of our experimental results. The result is $\tau=2.2 \times 10^{3}$ years for a grain on the surface of Europa and $\tau=0.7$ years on Dione. The calculated lifetimes demonstrate that the solid carbon can easily dissipate by conversion into $\mathrm{CO}_{2}$ and $\mathrm{CO}$ and finally become available for further radiolytic-induced processing.

Furthermore, structural and morphological changes in the icy and bare carbonaceous grains processed by ion irradiation with $200 \mathrm{keV} \mathrm{H}^{+}$were studied. Graphitization of the upper layer and graded processing of the carbon material in dependence on the penetration depth of the ions were observed. Less graphitization, characterized by longer graphene layers and more closed fullerene molecules/structures, compared with the original material were observed beneath the graphitic layer. The structural changes strongly affect the spectral properties of the carbonaceous material, especially in the far-IR.

The presented results based on bombardment experiments with $200 \mathrm{keV}$ protons also have consequences for the formation of more complex molecules in molecular clouds, protoplanetary disks, and the solar system and for recent dust models of the ISM (Draine 2009; Jones et al. 2013).

Acknowledgements. T.S. is supported by the European Community FP7-ITN Marie-Curie Program (LASSIE project, grant agreement No. 238258). We thank Gabriele Born (Astrophysikalisches Institut Jena) and Frank Steiniger (Universitaetsklinikum Jena) for their contributions in the sample preparation and FESEM diagnostics, respectively.

\section{References}

Baragiola, R. A. 2005, Nucl. Instr. Meth. Phys. Res. B, 232, 98

Baratta, G. A., Leto, G., Spinella, F., Strazzulla, G., \& Foti, G. 1991, A\&A, 252, 421

Bennett, C. J., Pirim, C., \& Orlando, T. M. 2013, Chem. Rev., 113, 9086

Bergin, E. A., Cleeves, L. I., Crockett, N., \& Blake, G. 2014, Faraday Discussions, 168, 61

Boduch, P., da Silveira, E. F., Domaracka, A., et al. 2011, Adv. Astron., 2011, 327641

Carlson, R. W., Anderson, M. S., Johnson, R. E., et al. 1999, Science, 283, 2062

Chiar, J. E., Adamson, A. J., Kerr, T. H., \& Whittet, D. C. B. 1994, ApJ, 426 240

Chiar, J. E., Adamson, A. J., Kerr, T. H., \& Whittet, D. C. B. 1995, News Letter Astron. Soc. New York, 4, 21

Chiar, J. E., Gerakines, P. A., Whittet, D. C. B., et al. 1998, ApJ, 498, 716

Clark, R. N., Carlson, R., Grundy, W., \& Noll, K. 2013, in The Science of Solar System ices, ed. M. Gudipati (Astrophysics and Space Science Library), 356, 3

de Graauw, T., Whittet, D. C. B., Gerakines, P. A., et al. 1996, A\&A, 315, L345 D’Hendecourt, L. B., Allamandola, L. J., \& Greenberg, J. M. 1985, A\&A, 152, 130

Draine, B. T. 2003, ARA\&A, 41, 241

Draine, B. T. 2009, in Cosmic Dust - Near and Far, eds. T. Henning, E. Grün, \& J. Steinacker, ASP Conf. Ser., 414, 453

Draine, B. T., \& Lee, H. M. 1984, ApJ, 285, 89

Ehrenfreund, P., Breukers, R., D'Hendecourt, L., \& Greenberg, J. M. 1992, A\&A, 260, 431

Ehrenfreund, P., Boogert, A. C. A., Gerakines, P. A., Tielens, A. G. G. M., \& van Dishoeck, E. F. 1997, A\&A, 328, 649

Ennis, C., Bennett, C. J., Jones, B. M., \& Kaiser, R. I. 2011, ApJ, 733, 79

Famá, M., Loeffler, M. J., Raut, U., \& Baragiola, R. A. 2010, Icarus, 207, 314

Fulvio, D., Sivaraman, B., Baratta, G. A., Palumbo, M. E., \& Mason, N. J. 2009, Spectrochim. Acta Part A: Molecular Spectroscopy, 72, 1007

Fulvio, D., Raut, U., \& Baragiola, R. A. 2012, ApJ, 752, L33

Gail, H.-P., Zhukovska, S. V., Hoppe, P., \& Trieloff, M. 2009, ApJ, 698, 1136

Gerakines, P. A., Schutte, W. A., \& Ehrenfreund, P. 1996, A\&A, 312, 289

Gerakines, P. A., Whittet, D. C. B., Ehrenfreund, P., et al. 1999, ApJ, 526, 1062

Gerakines, P. A., Moore, M. H., \& Hudson, R. L. 2000, A\&A, 357, 793

Gerakines, P. A., Moore, M. H., \& Hudson, R. L. 2001, J. Geophys. Res., 106, 33381

Gomis, O., \& Strazzulla, G. 2005, Icarus, 177, 570

Gomis, O., Satorre, M. A., Strazzulla, G., \& Leto, G. 2004, Planet. Space Sci., 52,371

Groenewegen, M. A. T., Waelkens, C., Barlow, M. J., et al. 2011, A\&A, 526 , A162 
Grundy, W. M., Young, L. A., \& Young, E. F. 2003, Icarus, 162, 222

Guertler, J., Henning, T., Koempe, C., et al. 1996, A\&A, 315, L189

Hendrix, A. R., Barth, C. A., \& Hord, C. W. 1999, J. Geophys. Res., 104, 14169

Henning, T., Michel, B., \& Stognienko, R. 1995, Planet. Space Sci., 43, 1333

Henning, T., Jäger, C., \& Mutschke, H. 2004, in Astrophysics of Dust, eds. A. N. Witt, G. C. Clayton, \& B. T. Draine, ASP Conf. Ser., 309, 603

Herbst, E. 2005, in ESA SP 577, ed. A. Wilson, 205

Hibbitts, C. A., Klemaszewski, J. E., McCord, T. B., Hansen, G. B., \& Greeley, R. 2002, J. Geophys. Res. (Planets), 107, 5084

Hibbitts, C. A., Pappalardo, R. T., Hansen, G. B., \& McCord, T. B. 2003, J. Geophys. Res. (Planets), 108, 5036

Hincelin, U., Wakelam, V., Hersant, F., et al. 2011, A\&A, 530, A6

Hudgins, D. M., Sandford, S. A., Allamandola, L. J., \& Tielens, A. G. G. M 1993, ApJS, 86, 713

Ioppolo, S., Palumbo, M. E., Baratta, G. A., \& Mennella, V. 2009, A\&A, 493, 1017

Ioppolo, S., van Boheemen, Y., Cuppen, H. M., van Dishoeck, E. F., \& Linnartz, H. 2011, MNRAS, 413, 2281

Ioppolo, S., Sangiorgio, I., Baratta, G. A., \& Palumbo, M. E. 2013, A\&A, 554, A34

Jäger, C., Mutschke, H., \& Henning, T. 1998, A\&A, 332, 291

Jäger, C., Mutschke, H., Henning, T., \& Huisken, F. 2008, ApJ, 689, 249

Jenkins, E. B. 2009, ApJ, 700, 1299

Jiang, G. J., Person, W. B., \& Brown, K. G. 1975, J. Chem. Phys., 62, 1201

Johnson, R. E., Famá, M., Liu, M., et al. 2008, Planet. Space Sci., 56, 1238

Jones, B. M., Bennett, C. J., \& Kaiser, R. I. 2011, ApJ, 734, 78

Jones, B. M., Gusmeroli, A., Arp, C. D., et al. 2013, International Journal of Remote Sensing, 34, 8267

Jones, B. M., Kaiser, R. I., \& Strazzulla, G. 2014, ApJ, 781, 85

Kerr, T. H., \& Adamson, A. J. 1993, in Astronomical Infrared Spectroscopy: Future Observational Directions, ed. S. Kwok, ASP Conf. Ser., 41, 219

Lamberts, T., Cuppen, H. M., Fedoseev, G., et al. 2014, A\&A, 570, A57

Lee, J.-E., Bergin, E. A., \& Nomura, H. 2010, ApJ, 710, L21

Leto, G., \& Baratta, G. A. 2003, A\&A, 397, 7

Li, A., \& Greenberg, J. M. 1997, A\&A, 323, 566

Loeffler, M. J., Baratta, G. A., Palumbo, M. E., Strazzulla, G., \& Baragiola, R. A. 2005, A\&A, 435, 587

Loeffler, M. J., Raut, U., Vidal, R. A., Baragiola, R. A., \& Carlson, R. W. 2006, Icarus, 180, 265

Madey, T. E., Johnson, R. E., \& Orlando, T. M. 2002, Surface Science, 500, 838

McCord, T. B., Carlson, R., Smythe, W., et al. 1997, Science, 278, 271

McCord, T. B., Hansen, G. B., Clark, R. N., et al. 1998, J. Geophys. Res., 103, 8603

Mennella, V., Colangeli, L., Palumbo, P., et al. 1996, in The Role of Dust in the Formation of Stars, eds. H. U. Käufl, \& R. Siebenmorgen, 317

Mennella, V., Palumbo, M. E., \& Baratta, G. A. 2004, ApJ, 615, 1073

Mennella, V., Baratta, G. A., Palumbo, M. E., \& Bergin, E. A. 2006, ApJ, 643, 923

Moore, M. H., \& Hudson, R. L. 1992, BAAS, 24, 1042

Moore, M. H., \& Hudson, R. L. 2000, Icarus, 145, 282

Moore, M. H., Khanna, R., \& Donn, B. 1991, J. Geophys. Res., 96, 17541

Moore, M. H., Ferrante, R. F., \& Nuth, III, J. A. 1996, Planet. Space Sci., 44, 927
Moore, M. H., Hudson, R. L., \& Gerakines, P. A. 2001, Spectrochim. Acta, 57, 843

Noble, J. A., Dulieu, F., Congiu, E., \& Fraser, H. J. 2011, ApJ, 735, 121

Palumbo, M. E., \& Baratta, G. A. 2000, A\&A, 361, 298

Palumbo, M. E., \& Strazzulla, G. 1993, A\&A, 269, 568

Palumbo, M. E., Baratta, G. A., Brucato, J. R., et al. 1998, A\&A, 334, 247

Peeters, Z., Hudson, R. L., Moore, M. H., \& Lewis, A. 2010, Icarus, 210, 480

Pilling, S., Seperuelo Duarte, E., Domaracka, A., et al. 2010, A\&A, 523, A77

Pontoppidan, K. M., Boogert, A. C. A., Fraser, H. J., et al. 2008, ApJ, 678, 1005

Porter, S. B., Desch, S. J., \& Cook, J. C. 2010, Icarus, 208, 492

Raut, U., \& Baragiola, R. A. 2011, ApJ, 737, L14

Raut, U., Loeffler, M. J., Famá, M., \& Baragiola, R. A. 2011, J. Chem. Phys., 134, 194501

Raut, U., Fulvio, D., Loeffler, M. J., \& Baragiola, R. A. 2012, ApJ, 752, 159

Sabri, T., Gavilan, L., Jäger, C., et al. 2014, ApJ, 780, 180

Sandford, S. A., \& Allamandola, L. J. 1990, Icarus, 87, 188

Sandford, S. A., Allamandola, L. J., Tielens, A. G. G. M., \& Valero, G. J. 1988 ApJ, 329, 498

Satorre, M. A., Palumbo, M. E., \& Strazzulla, G. 2000, Ap\&SS, 274, 643

Smith, M. A. H., Rinsland, C. P., Fridovich, B., \& Rao, K. N. 1985, in Molecular Spectroscopy: Modern Research, ed. K.N. Rao, Vol. 3 (New York: Academic Press), 112

Stognienko, R., Henning, T., \& Ossenkopf, V. 1995, A\&A, 296, 797

Strazzulla, G. 2011, Nucl. Instr. Meth. Phys. Res. B, 269, 842

Strazzulla, G., Leto, G., \& Palumbo, M. E. 1993, Adv. Space Res., 13, 189

Strazzulla, G., Brucato, J. R., Palumbo, M. E., \& Satorre, M. A. 1997, A\&A 321,618

Strazzulla, G., Baratta, G. A., \& Palumbo, M. E. 2001, Spectrochim. Acta, 57 825

Teixeira, T. C., Emerson, J. P., \& Palumbo, M. E. 1998, A\&A, 330, 711

Teolis, B. D., \& Waite, J. H. 2012, AGU Fall Meeting Abstracts, A2

Teolis, B. D., Jones, G. H., Miles, P. F., et al. 2010, Science, 330, 1813

Tielens, A. G. G. M., Tokunaga, A. T., Geballe, T. R., \& Baas, F. 1991, ApJ, 381, 181

Vandenbussche, B., Ehrenfreund, P., Boogert, A. C. A., et al. 1999, A\&A, 346, L57

Watanabe, N., \& Kouchi, A. 2002, ApJ, 567, 651

Whittet, D. C. B. 1993, in Observations of Molecular Ices, eds. T. J. Millar, \& D. A. Williams, 9

Whittet, D. C. B. 2010, ApJ, 710, 1009

Whittet, D. C. B., \& Walker, H. J. 1991, MNRAS, 252, 63

Whittet, D. C. B., Gerakines, P. A., Tielens, A. G. G. M., et al. 1998, ApJ, 498, L159

Whittet, D., Bergin, E., Chiar, J., et al. 2007, Spitzer Proposal, 40432

Yamada, H., \& Person, W. B. 1964, J. Chem. Phys., 41, 2478

Zheng, W., \& Kaiser, R. I. 2007, Chem. Phys. Lett., 450, 55

Zheng, W., Jewitt, D., \& Kaiser, R. I. 2006, ApJ, 648, 753

Zhukovska, S., \& Gail, H.-P. 2007, in Why Galaxies Care About AGB Stars: Their Importance as Actors and Probes, eds. F. Kerschbaum, C. Charbonnel, \& R. F. Wing, ASP Conf. Ser., 378, 499

Zhukovska, S., \& Henning, T. 2013, A\&A, 555, A99

Ziegler, J. F., Ziegler, M. D., \& Biersack, J. P. 2010, Nucl. Instr. Meth. Phys. Res. B, 268, 1818 